\title{
Ocular complications in newly diagnosed borderline lepromatous and lepromatous leprosy patients: baseline profile of the Indian cohort
}

\author{
E Daniel, S Koshy, G Sundar Rao, P S S S Rao
}

Series editors: W V Good and S Ruit

See end of article for authors' affiliations

Correspondence to: Dr Ebenezer Daniel Department of Ophthalmology, Schieffelin Leprosy Research and Training Center, Karigiri, Vellore District, Tamilnadu, India - 632106;

oculep@vsnl.com

Accepted for publication 3 July 2002

\begin{abstract}
Aim: To describe ocular manifestations in newly diagnosed borderline lepromatous (BL) and lepromatous leprosy (LL) patients in India.

Methods: Ocular complications, at enrolment, occurring in all new borderline lepromatous and lepromatous leprosy patients detected by active case finding within the geographically defined leprosy endemic area of the Gudiyattam Taluk in India from 1991 to 1997 who consented to ocular examinations every 6 months, during and 5 years after treatment with multidrug therapy (MDT), were studied. Results: Orbicularis oculi weakness $(4.62 \%)$, lagophthalmos $(4.20 \%)$, ectropion $(0.42 \%)$, trichiasis $(0.84 \%)$, blocked nasolacrimal ducts (1.68\%), pterygium (1 1.34\%), impaired corneal sensation (53\%), corneal opacity $(10.5 \%)$, corneal nerve beading $(1.68 \%)$, punctate keratitis $(1.26 \%)$, keratic precipitates $(4.62 \%)$, iris atrophy $(1.68 \%)$, and cataract $(12.6 \%)$ were ocular complications seen in the 301 lepromatous patients at enrolment. $4.6 \%$ had blind eyes. Increasing age was associated with ocular complications. $80 \%$ of patients were skin smear acid fast bacilli (AFB) positive. The LL/BL ratio was 1:6.4. $71 \%$ had some limb deformity. $44 \%$ had only leprosy related ocular complications (LROC), $28 \%$ had only general ocular complications (GOC) while $14 \%$ had both LROC and GOC. Ocular complications were significantly related to leg deformities. Corneal nerve beading was seen most in LL patients (100\%) having high bacterial content. Lagophthalmos and muscle weakness were associated with reversal reactions.

Conclusions: Corneal nerve beading occurs in LL patients with high bacillary count. Patients with reversal reaction are more likely to present with orbicularis oculi weakness and lagophthalmos. Leprosy related ocular complications and general ocular complications are significant problems in newly diagnosed lepromatous patients. Elderly, deformed, skin smear positive, lepromatous patients are associated with increased ocular morbidity and form a group that require acceptable and accessible eye care.
\end{abstract}

G erald Henrik Armauer Hansen, credited with the discovery of Mycobacterium leprae had said "There is no disease which so frequently gives rise to disorders of the eye, as leprosy does." ' Leprosy, at that time, was spread throughout the world with no effective treatment. Similar to many other infectious diseases, leprosy has undergone drastic changes in the past two decades. The introduction of multidrug therapy (MDT) in the early 1980s has begun to have an impact on the transmission of the disease and the severity of its attending complications. Among the serious complications that accompany this disease is that of ocular morbidity and blindness. Many ocular surveys have been done on ocular leprosy, most of them in the pre-MDT era. ${ }^{2-8}$ The reported prevalence of ocular complications in leprosy varies from $6 \%$ to $96 \%$. The reasons for this wide variation are lack of survey sampling techniques, using non-ophthalmologically trained individuals as examiners, failure to analyse by leprosy characteristics, lack of adequate instrumentation to detect ocular signs and not clearly defining the ocular complications. ' Most prevalence studies have been done in leprosy hospitals or in eye clinics and suffer from selection bias with a possible overestimation of ocular complications. There are no studies on incident ocular complications in leprosy in India, one of the few countries in the world in which leprosy is still endemic despite intensive efforts to eliminate the disease. Eighty per cent of the world's 12 million MDT cured leprosy patients reside in India and around 600000 new patients are diagnosed annually. Multibacillary (MB) leprosy patients are known to get more ocular complications than the paucibacil- lary (PB) patients. ${ }^{10}{ }^{11}$ Recognising the need to document the magnitude of ocular complications in newly diagnosed leprosy patients and investigate the risk factors for ocular involvement and blindness in these patients, a multicentre study called "The longitudinal Study of Ocular Leprosy (LOSOL)" was formulated. One of the study sites was located in the Indian subcontinent with the aim of recruiting a cohort of newly diagnosed MB patients and following them up during and after treatment with MDT. Recruitment of all newly diagnosed lepromatous patients was started in 1991 and completed in 1997. In this paper we describe the demographic, leprosy related, and ocular baseline profile of patients recruited in the Indian cohort study.

\section{METHODS}

The Gudiyattam Taluk is a geographically defined area of 1306 square kilometres situated in the Vellore district of Tamilnadu, south India, with an estimated population of 650000 . This region was one of the highest endemic areas for leprosy in India with more than 2000 new cases detected annually in the 1960s, and even now it has not reached the World Health

Abbreviations: AFB, acid fast bacilli; $\mathrm{BI}$, bacteriological index; $\mathrm{BL}$, borderline lepromatous; GOC, general ocular complications; LL, lepromatous leprosy; LROC, leprosy related ocular complications; $M B$, multibacillary; MDT, multidrug therapy; PB, paucibacillary; PCT, pupil cycle time 
Table 1 Sex and age groups of newly diagnosed lepromatous patients

\begin{tabular}{lccc}
\hline Age groups & Male & Female & Total \\
\hline $1-20$ years & 16 & 3 & 19 \\
$21-30$ years & 27 & 12 & 39 \\
$31-40$ years & 45 & 19 & 64 \\
$41-50$ years & 23 & 22 & 45 \\
$51-60$ years & 31 & 9 & 40 \\
61 years and above & 25 & 6 & 31 \\
Total & 167 & 71 & 238 \\
\hline
\end{tabular}

Organization elimination target of less that one leprosy patient per 10000 population. Until 1997, for over 35 years, the Schieffelin Leprosy Research and Training Centre (SLRTC) had a leprosy control programme in this area that actively recruited patients for treatment with MDT. From 1991 to 1997 all newly diagnosed $\mathrm{MB}$ patients were invited to form a cohort in order to study the prevalence and incidence of ocular complications occurring in these patients during and after MDT. The patients were required to come for a comprehensive ocular examination once every 6 months, both during therapy with the 2 year MDT and during a 5 year period after being released from treatment. Ocular examinations were to be done by an experienced ophthalmologist and results recorded in an eye examination sheet. All MB patients accepted enrolment in the study after due informed consent was obtained except for 13 male and two female patients who refused to take part in the study.

After leprosy diagnosis was made in the field, patients were sent to SLRTC where they were examined and the disease documented by experienced leprologists. The leprosy data on each patient included the type of leprosy according to both the $\mathrm{WHO}^{12}$ and the Ridley and Jopling ${ }^{13}$ classification, the approximate duration of the disease calculated in years from the time of the appearance of the first symptom to the time of enrolment, the bacteriological index (BI) from skin smear examination of routine and special sites, the deformity status graded according to the WHO criteria, ${ }^{14}$ history and the presence of reversal reactions (RR) or erythema nodosum leprosum (ENL) and the presence or absence of a face patch. The duration of the disease was counted as 1 year for patients with duration of 1 year or less. Face patch was defined as any hypopigmented or erythematous patch, big or small, occurring anywhere over the face. Ocular examination consisted of first estimating visual acuity with a Snellen chart. The presence of lagophthalmos, measurements of the maximum palpebral width on gentle and forceful closure of the lids if lagophthalmos was present, the presence of orbicularis oculi muscle weakness estimated by gently pulling down on the lower lid on forceful closure, presence of ectropion, entropion, trichiasis, patency of the nasolacrimal duct determined by syringing the duct and the presence of conjunctivitis, conjunctival scarring, episcleritis, or scleritis were docu- mented. Presence of clofazamine crystals in the cornea or conjunctiva, corneal opacities, pterygium, corneal vascularisation, corneal nerve beading, punctate keratitis, and corneal ulcers were noted. Corneal sensation was estimated by asking the patient to look up and applying the tail end of a wisp of cotton on the cornea $2 \mathrm{~mm}$ from the limbus at the 6 o'clock position and categorising the sensation as normal if the patient responded by retracting the head or shutting the eyelids and impaired if the patient did not. Presence of keratic precipitates, flare, and cells in the anterior chamber, circumcorneal congestion, and the pupil shape and size were recorded. Intraocular tension was estimated using a Goldmann applanation tonometer and the presence of any opacities in the lens was recorded. Pupil cycle time (PCT) was estimated using the technique already described. ${ }^{15}$

STATA 7.0 was used for the statistical analysis. Ocular complications were categorised into leprosy related ocular complications (LROC) and general ocular complications (GOC). LROC consisted of orbicularis oculi weakness, lagophthalmos, ectropion, entropion, trichiasis, episcleritis, scleritis, clofazimine crystals, impaired corneal sensation in both eyes, corneal opacity associated with decrease in visual acuity, corneal ulcer, corneal nerve beading, punctate keratitis, episcleritis, scleritis, iridocyclitis, iris atrophy, and cataract with decreased vision associated with keratic precipitates or flare and cells. GOC consisted of patients with nasolacrimal duct blockage, pterygium, and age related cataract with visual acuity of $6 / 18$ or less. Analysis was done by people rather than by eyes. $\chi^{2}$ Tests were used for analysis of categorical variable and simple regression for continuous variables. The independent effect of risk factors was evaluated with logistic regression models.

\section{PARTICIPANTS}

\section{Demographic characteristics}

A total of 238 lepromatous patients were enrolled, 167 (70\%) male and 71 (30\%) female. There was no significant difference in the occurrence of ocular complications between sexes.

Age of patients ranged from 7 to 78 years with a mean of 41.15 (SD 14.90) years and a median of 40 years. The sex and age groups of the newly diagnosed patients is given in Table 1. Age was significantly associated with decreased vision of 6/18 or worse $(p<0.001)$, blindness in any one eye $(p<0.001)$, hand deformity $(p=0.003)$, leg deformity $(p<0.001)$, blocked nasolacrimal duct $(p=0.009)$, pterygium $(p<0.001)$, PCT above $800 \mathrm{~ms}(\mathrm{p}=0.025)$, impaired corneal sensation in one or both eyes $(p=0.004)$, corneal sensory impairment in both eyes $(p=0.011)$, keratic precipitates $(p=0.002)$, cataract $(p<0.001)$, and intraocular pressure less than $8 \mathrm{~mm} \mathrm{Hg}$ $(p=0.048)$. Thirty two patients had predominantly indoor occupations while the rest had outdoor occupations. Type of occupation was not significantly associated with any of the independent variables.

\section{Leprosy characteristics}

There were 32 lepromatous leprosy (LL) patients and 206 borderline lepromatous (BL) patients making a LL:BL ratio of $1: 6.4$.

Table 2 Limb deformity status of the cohort at enrolment, graded according to the recent $\mathrm{WHO}$ classification

\begin{tabular}{llll}
\hline \multirow{2}{*}{ Limb } & No deformity & Grade I deformity & Grade II deformity \\
\cline { 2 - 4 } & No $(\%)$ & No $(\%)$ & No $(\%)$ \\
\hline Right hand & $131(55)$ & $62(26)$ & $45(19)$ \\
Leff hand & $136(57)$ & $63(27)$ & $39(16)$ \\
Right leg & $100(42)$ & $106(45)$ & $32(13)$ \\
Left leg & $99(42)$ & $118(49)$ & $21(9)$ \\
\hline
\end{tabular}


Table 3 Visual acuity of patients at enrolment

\begin{tabular}{|c|c|c|c|c|c|}
\hline & \multicolumn{5}{|l|}{ Vision } \\
\hline & $6 / 6-6 / 12$ & $6 / 18$ or less & $6 / 60$ or less & $<3 / 60$ & $\begin{array}{l}<3 / 60 \text { in both } \\
\text { eyes }\end{array}$ \\
\hline No of patients & 204 & 34 & 17 & 11 & 2 \\
\hline Percentage & 85.7 & 14.3 & 7.1 & 4.6 & 0.84 \\
\hline
\end{tabular}

History of having had a face patch occurred more often in BL than in LL $(p<0.001)$ patients. LL patients had more deformities $(p=0.01)$, more bacilli $(p<0.001)$, fewer reactions $(\mathrm{p}=0.012)$, and more pterygia $(\mathrm{p}=0.043)$ than BL patients. There was a significant association between corneal nerve beading and lepromatous leprosy $(\mathrm{p}<0.000)$, all four patients who had corneal nerve beading belonged to the LL group. Duration of disease ranged from 1 to 30 years with mean of 3.3 (SD 5.4) years. In all, 47 (20\%) patients were skin smear negative for acid fast bacilli (AFB) at enrolment. The average BI ranging from 0 to 5.5 with a mean of 1.4 (1.4). Highest skin smear for AFB at any one site ranged from 0 to 6 with a mean of 2.2 (1.8). Patients with more than 1.00 average BI, patients were associated with orbicularis oculi muscle weakness $(\mathrm{p}=0.035)$, lagophthalmos $(\mathrm{p}=0.05)$, and corneal nerve beading $(\mathrm{p}=0.047)$.

Limb deformity, categorised according to the recent WHO classification, ${ }^{16}$ in patients at enrolment is given in Table 2 . Sixty eight $(28.5 \%)$ patients had grade 0 deformity (no deformity) in any of the limbs; grade 2 deformity in all the limbs were found in six $(2.5 \%)$ patients. Deformities were significantly associated with age $(p=0.001)$. Trichiasis $(p<0.001)$ was significantly more in patients who had grade 2 deformity in all the limbs. A smaller percentage of LL patients $(9.3 \%)$ had no deformities in any limb compared with BL patients $(31 \%)(p=0.01)$.

Diabetes mellitus co-existed in two patients while tuberculosis was seen in one patient. Thirty six per cent of the patients gave a history of having had either a erythematous lesion or a hypopigmented lesion in the face. More women $(51 \%)$ than men $(30 \%)(p<0.001)$, more BL patients $(p<0.001)$, and patients with shorter duration of disease $(p=0.01)$ gave a history of face patch. History of face patch was also associated with orbicularis oculi weakness $(p=0.05)$ and lagophthalmos $(\mathrm{p}=0.02) ; 42 \%$ of patients presented with face patch at enrolment and except for one patient all belonged to the BL type $(p<0.001)$. The presentation of face patches at enrolment was

Table 4 Ocular complications in newly diagnosed lepromatous patients

\begin{tabular}{lcc}
\hline Ocular complications & $\begin{array}{l}\text { No of patients } \\
(238)\end{array}$ & Percentage \\
\hline Lagophthalmos & 10 & 4.20 \\
Ectropion & 1 & 0.42 \\
Entropion & 0 & 0.00 \\
Trichiasis & 2 & 0.84 \\
Episcleritis & 1 & 0.42 \\
Scleritis & 0 & 0.00 \\
Corneal opacity & 25 & 10.50 \\
Corneal nerve beading & 4 & 1.68 \\
Punctate keratitis & 3 & 1.26 \\
Corneal ulcer & 0 & 0.00 \\
AC flare and cells & 1 & 0.42 \\
Keratic precipitates & 11 & 4.62 \\
Iris atrophy & 4 & 1.68 \\
Cataract & 57 & 24.00 \\
Pterygium & 27 & 11.34 \\
Blocked nasolacrimal duct & 4 & 1.68 \\
\hline
\end{tabular}

associated with female sex $(p=0.048)$. Fourteen per cent of patients gave history of reactions and were associated with more hand deformities $(p=<0.001)$, leg deformity $(p=0.03)$ and deformity in any limbs $(p=0.002)$. History of reactions were not associated with lagophthalmos and orbicularis weakness $(p>0.05) ; 24 \%$ had reversal reaction at enrolment that was associated with BL $(p=0.012)$, more than $1.00 \mathrm{BI}$ at any one site $(p=0.009)$, orbicularis weakness $(p<0.001)$, and lagophthalmos $(\mathrm{p}<0.001)$. The odds ratio of having a lagophthalmos was 8.3 (SE 5.88) with $95 \%$ CI of 2.07 to 33 ( $p=0.003$ ) when the patient presented with a type I reaction.

\section{Ocular characteristics}

The visual acuity of patients in any or both eyes at enrolment is given in Table 3. Only two patients were blind when the WHO definition of blindness was applied. Ocular complications seen at enrolment and measured ocular involvements are listed in Tables 3 and 4.81 patients $(34 \%)$ had neither LROC nor GOC, 20 (8.4\%) had only GOC, 104 (43.7\%) had only LROC, and 33 (13.9\%) patients had both LROC and GOC. LROC with inclusion of impaired corneal sensation was significantly associated with increasing age $(p=0.005)$. Visual loss and blindness were not significantly related to LROC $(p>0.05)$. LROC without the inclusion of impaired corneal sensation (LROC-CORSEN) was significantly associated with age $(p<0.001)$, increased average BI $(p=0.03)$, increase in BI at any one site $(p=0.008)$, hand deformity $(p=0.002)$, leg deformity $(p=0.001)$, grade II deformity in all limbs $(p=0.010)$, decreased vision $(p<0.001)$, and blindness $(p<0.001)$. PCT of more than $800 \mathrm{~ms}$ in both eyes $(\mathrm{p}=0.027)$ and age related cataract with and without decreased vision $(p<0.001)$ were significantly associated with LROC-CORSEN. When all these variables were included in a multiple regression model, age related cataracts $(p<0.001)$ and decreased vision $(p=0.004)$ remained significantly associated with LROC. GOC was associated with increasing age $(p<0.001)$, leg deformities $(p=0.002)$, deformities in general $(p=0.034)$, decreased vision $(p<0.000)$, and blindness $(p<0.000)$. After controlling for age only the association of decreased vision and blindness remained significant.

Combined leprosy related and general complications (ALLCOMP) were associated with increasing age $(p<0.001)$, leg deformity $(\mathrm{p}<0.008)$, any deformity $(\mathrm{p}=0.007)$, decreased vision $(p<0.000)$ and blindness $(p<0.000)$. Controlling for age, only decreased vision was significant. Intraocular pressure over $20 \mathrm{~mm} \mathrm{Hg}$ was not associated significantly with any variable. PCT of less than $650 \mathrm{~ms}$ in any one eye was significantly associated with younger age group $(p=0.05)$. PCT of less than $650 \mathrm{~ms}$ in both eyes were significantly associated with reversal reaction $(p=0.006)$. PCT of more than $800 \mathrm{~ms}$ in any one eye were significantly associated with leg deformities $(p=0.03)$. PCT of more than $800 \mathrm{~ms}$ in both eyes were significantly associated with older age group $(p=0.025)$, leg deformity $(p=0.04)$, flare and cells in the anterior chamber $(p=0.02)$, and iris atrophy $(\mathrm{p}=0.057)$.

\section{DISCUSSION}

\section{Demographic and leprosy characteristics}

Ageing was significantly associated with increasing LROC, GOC, and vision loss. Although an expected finding, this feature has 
Table 5 Other ocular involvement in lepromatous patients at enrolment

\begin{tabular}{lll}
\hline Ocular measurements & $\begin{array}{l}\text { No of patients } \\
(238)\end{array}$ & Percentage \\
\hline Orbicularis oculi weakness & 11 & 4.62 \\
Lagophthalmos with $>2$ mm on gentle closure & 5 & 2.10 \\
Lagophthalmos with palpebral gap on forced closure & 4 & 1.68 \\
Impaired corneal sensation in one eye & 173 & 72.69 \\
Impaired corneal sensation in both eyes & 127 & 53.36 \\
Corneal opacity with reduced vision & 4 & 1.68 \\
PCT $>800$ ms in one or both eyes & 53 & 27.75 \\
PCT $>800$ ms in both eyes & 30 & 15.71 \\
IOP $>20$ mm Hg & 5 & 2.12 \\
IOP $<8$ mm Hg & 4 & 1.68 \\
Cataract associated with $\leqslant 6 / 18$ vision & 30 & 12.61 \\
Cataract associated with uveitis & 5 & 2.10 \\
\hline
\end{tabular}

not been explicitly highlighted in most studies. As leprosy populations become older, their age related complications together with their ocular morbidity and persisting stigma to the disease come together to create a situation where the need to put in place accessible healthcare facilities becomes critical. Patients with grade II deformity in all limbs have more trichiasis and patients with one or more grades of disability in one or more limbs have more LROC as well as GOC than those patients who have no deformity in any of the limbs. This clearly demarcates a group of patients who have more limb deformities and more ocular complications. Stigma to leprosy has been shown to have a strong association with limb deformity. Therefore, this group of most disadvantaged are the ones who that need the most health care but have the least access to that care. A unusual finding was the presence of a significant association between ocular complications and leg deformities and absence of such an association with hand deformities for both LROC and GOC. This association disappeared after controlling for age.

One third of the patients gave a history of having had either an erythematous or a hypopigmented face patch. Recall bias may be a reason for its occurrence in more women and patients with shorter duration of the disease. An earlier study had placed great emphasis on the association of face patches in the zygomatic area or over the lids with lagophthalmos in paucibacillary leposy ${ }^{17}$ and we also found such an association in our multibacillary group. However, in our study, face patches were defined as any hypopigmented or erythematous patches that occurred over the face and not just patches which overlay the zygomatic area or spread over the eyelids. History of type I reactions were associated with lagophthalmos but type I reactions at enrolment were. Patients presenting with a type I reaction were eight times more likely to get lagophthalmos than those who did not have type I reaction. Borderline lepromatous patients with type I reaction were more likely to get orbicularis muscle weakness or lagophthalmos. The borderline type of the disease with an upgrading reaction points towards a resurgence of cell mediated immunity that may be deleterious to the superficial nerves in the body which includes the zygomatic branch of the facial nerve.

Eighty per cent of patients in this cohort had demonstrable $M$ leprae in their skin smears at enrolment as is to be expected from a group of lepromatous patients. Patients with higher skin smear counts of $M$ leprae, in individual sites or as average bacterial indices, were associated with more orbicularis oculi weakness and lagophthalmos. In the lepromatous group of patients (BL and LL), having a higher number of mycobacteria may be a risk factor for the development of lagophthalmos and orbicularis oculi muscle weakness. Corneal nerve beading was found only in $2 \%$ of patients but was exclusively seen in LL patients. There was also a significant association between corneal nerve beading and a high mycobacterial count in skin smears. Corneal nerve beading was not associated with features of intraocular inflammation. If biomicroscopic examination reveals corneal nerve beading there is a very high probability that the patient is an LL with a high bacillary count.

\section{Ocular characteristics}

The prevalent ocular complications listed in Table 5 are important reminders that although MDT has been extremely successful in treating leprosy in many endemic countries, a good proportion of the patients may present with pre-existing ocular morbidity. The present study has been done in a country that has the highest prevalence and incidence of leprosy in the world but the geographical region chosen for the study has been one in which a proficient leprosy control programme with active case finding has been in existence for several decades. Therefore, these data may underestimate ocular complications prevalent in other parts of the country where intensive leprosy control programmes are absent and where other detrimental factors to accessing health care exist. LROC was present in $58 \%$ of patients. If impaired corneal sensation, a substantially subjective complication that is difficult to estimate consistently, ${ }^{18}$ was excluded in LROC, then this figure falls to $23 \%$. Elderly, deformed, and lepromatous patients with AFB positive skin smears and prolonged $\mathrm{PCT}^{19}$ are associated with LROC. GOC showed a similar association with leg deformities but when controlling for age this association was lost. GOC also occurred more frequently in LL than in BL patients but this association was also primarily due to age rather than being an LL patient. Age related cataract was the main individual cause of vision loss in this cohort and will increasingly contribute to ocular morbidity in an ageing leprosy population. This is especially true in a country like India that has the highest number of age related cataract in the world coupled with the largest number of leprosy patients. Leprosy patients are still hampered with the stigma attached to the disease and seeking access for surgical correction of their cataracts may be a difficult task, especially in areas where leprosy is still endemic and good healthcare support scanty.

This cohort of lepromatous patients recruited actively over a period of 7 years in an endemic area that has not yet reached elimination status provides proof that newly diagnosed lepromatous patients present with significant numbers of limb deformities, leprosy related ocular complications, and general ocular complications. This has wide ranging implications for health policy issues when integration of vertical leprosy control programmes into general health programmes, a factor that is rapidly gaining momentum in leprosy endemic nations, is taken into account. Increasingly the primary healthcare (PHC) centres and district hospitals are required to take up responsibility of diagnosing leprosy and its attending complications, administering MDT and in treating all complications, leprosy related or otherwise, that occur in these patients. Newly diagnosed incident cases of leprosy are purported to be around 600000 in 
India and it is likely that a considerable number of them would be lepromatous (LL and BL) patients. The health facilities that have been designated to deal with these patients must have personnel that have reasonably adequate knowledge and basic instrumentation to deal with the ocular complications that are likely to present in these patients.

\section{ACKNOWLEDGEMENTS}

We are grateful to Dr Paul Courtright, Dr Timothy ffytche, and Mr P Yowan for their assistance with this study. This project was supported by Lepra, Colchester, UK.

\section{Authors' affiliations}

E Daniel, S Koshy, P S S S Rao, Department of Ophthalmology,

Schieffelin Leprosy Research and Training Center, Karigiri, Vellore

District, Tamilnadu, India - 632106

G Sundar Rao, Department of Community Health

\section{REFERENCES}

1 ffytche T. Blindness in leprosy-a forgotten complication. Aust NZ J Ophthalmol 1989;17:257-60.

2 Narain M, Mukhija RD, Bist HK, et al. A study of ocular complications in leprosy. Indian J Lepr 1984;56:241-50.

3 Rawal RC, Kar PK, Desai RN, et al. A clinical study of eye complications in leprosy. Indian J Lepr 1984;56:232-40.

4 Reddy SC, Raju BD, Achary NR. Survey of eye complications in leprosy in Prakasam District (Andhra Pradesh). Lepr India 1981;53:231-7.

5 Acharya BP. Ocular involvement in leprosy (a study in mining areas of India). Indian J Ophthalmol 1978;26:21-4.
6 Balakrishnan E. Survey of ocular complications in lepromatous leprosy J All India Ophthalmol Soc 1966;14:214-6.

7 Dutta LC, Das NC, Chatteriee BC, et al. Ocular lesions in leprosy. J Indian Med Assoc. 1973;61:385-8.

8 Joshi PB, Shah AH, Agashe PK, et al. Ocular manifestations of leprosy. Indian J Med Res 1973;61:435-41.

9 Courtright PD. Defining the magnitude of ocular complications from leprosy: problems of methodology. Int J Lepr Other Mycobact Dis 1988;56:566-73

10 Malla OK, Brandt F, Anten JG. Ocular findings in leprosy patients in an institution in Nepal (Khokana). Br J Ophthalmol 1981;65:226-30.

11 Soshamma G, Suryawanshi N. Eye lesions in leprosy. Lepr Rev 1989:60:33-8.

12 World Health Organization. Report of a study group: chemotherapy of leprosy for control programmmes. WHO Tech Rep Ser 1982:675.

13 Ridley DS, Jopling WH. Classification of leprosy according to immunity - a five group system. Int J Lepr Other Mycobact Dis 1966;34:255-73.

14 WHO Expert Committee on Leprosy. Sixth Report. WHO Tech Rep Ser 1988:768.

15 Miller SD, Thompson HS. Edge-light pupil cycle time. Br J Ophthalmol 1978:62:495-500.

16 WHO Expert Committee on Leprosy. Sixth report. Geneva: WHO Tech Rep Ser 1988:768.

17 Hogeweg M, Kiran K, Suneetha S. The significance of facial patches and type I reaction for the development of facial nerve damage in leprosy. A retrospective study among 1226 paucibacillary leprosy patients. Lepr Rev 1991;62:143-9.

18 Daniel E. Corneal sensation in leprosy. Int J Lepr Other Mycobact Dis 1999:67:298-301.

19 Daniel E, Rao PSS. Pupil cycle time in leprosy patients without clinically apparent ocular pathology. Int $J$ Lepr Other Mycobact Dis 1995;63:529-34.pick;bjo.cam.f1;0;1

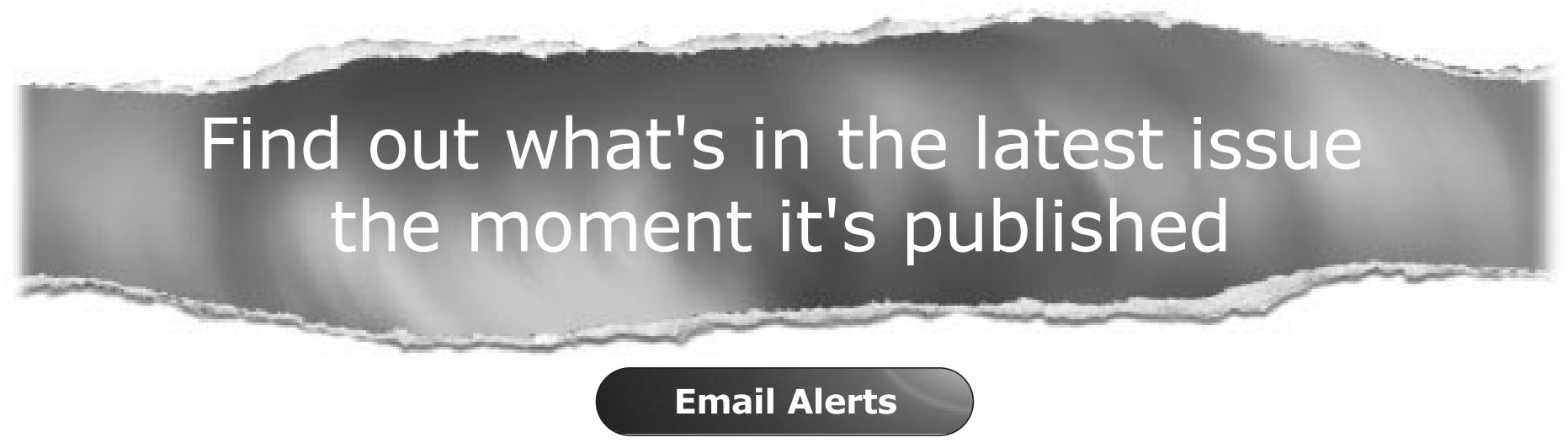

Sign up to receive the table of contents by email every month. You can select from three alerts: Table of Contents (full), TOC Awareness (notice only); British Journal of Ophthalmology related announcements.

\section{www.bjophthalmol.com}

